\title{
Gut-brain communication and obesity: understanding functions of the vagus nerve
}

\author{
Hans-Rudolf Berthoud, ${ }^{1}$ Vance L. Albaugh, ${ }^{2}$ and Winfried L. Neuhuber ${ }^{3}$ \\ ${ }^{1}$ Neurobiology of Nutrition and Metabolism Department and ${ }^{2}$ Bariatric and Metabolic Institute, Pennington Biomedical Research Center, Louisiana State University, Baton Rouge, Louisiana, USA. ${ }^{3}$ Institute \\ for Anatomy and Cell Biology, Friedrich-Alexander University, Erlangen, Germany.
}

\begin{abstract}
Given the crucial role of the gastrointestinal tract and associated organs in handling nutrient assimilation and metabolism, it has long been known that its communication with the brain is important for the control of ingestive behavior and body weight regulation. It is also clear that gut-brain communication is bidirectional and utilizes both rapid neural and slower humoral mechanisms and pathways. However, progress in understanding these mechanisms and leveraging them for the treatment of obesity and metabolic disease has been hindered by the enormous dimension of the gut mucosa, the complexity of the signaling systems, and lack of specific tools. With the ascent of modern neurobiological technology, our understanding of the role of vagal afferents in gut-brain communication has begun to change. The first function-specific populations of vagal afferents providing nutritional feedback as well as feed-forward signals have been identified with genetics-guided methodology, and it is hoped that extension of the methodology to other neural communication pathways will follow soon. Currently, efficient clinical leveraging of gut-brain communication to treat obesity and metabolic disease is limited to a few gut hormones, but a more complete understanding of function-specific and projection-specific neuronal populations should make it possible to develop selective and more effective neuromodulation approaches.
\end{abstract}

\section{Introduction}

Given that the prevalence of obesity is approaching $50 \%$ in some countries (1), it is hard to argue that body weight/adiposity is regulated at healthy levels through biological mechanisms (2-7). It has been speculated that the best explanation for this conundrum is evolutionary genetic drift (7) and the dependency of such regulation on the environment and lifestyle $(8,9)$. In genetically susceptible individuals, pressures from food, physical, and social environments (10) seem to be too much for a regulatory system that evolved mainly to defend against starvation $(7,11)$. Being unable to change the root cause, namely an environment that facilitates overnutrition and discourages physical activity, the fight against obesity is currently limited to using known physiological systems controlling energy intake, assimilation, and expenditure for behavioral, pharmacological, and surgical therapies. As such, the gut-brain axis is a key element for the control of ingestive behavior with important implications for the development of obesity and metabolic diseases. Here we discuss recent advances in understanding how nutrients are perceived by the gastrointestinal tract and how relevant signals are mediated to the brain, with emphasis on the vagus nerve.

\section{The bases of bidirectional gut-brain crosstalk} Although use of the term "gut-brain axis" on PubMed has increased more than tenfold only in the last decade, it has long been known that connections between these organs exist and

Conflict of interest: The authors have declared that no conflict of interest exists. Copyright: (5) 2021, American Society for Clinical Investigation.

Reference information: / Clin Invest. 2021;131(10):e143770.

https://doi.org/10.1172/JCl143770. that they have important functional implications in health and disease. For example, Galenus of Pergamum, a surgeon and philosopher in the Roman Empire (129-199 CE), noted: "A large portion of nerves is emitted from the brain to the entrance of the stomach, because nature has made this an instrument of appetite for food, which is at the door ... of all instruments nature has prepared for management of nutrients" (translated from ref. 12). The recent fascination with the term is almost solely due to literature on the gut microbiome (e.g., ref. 13), covered in other articles in this Review series. However, for students of ingestive behavior and metabolic physiology, understanding the gut-brain axis has been a crucial element for half a century (e.g., ref. 14). It was this interest in ingestive behavior and the rising obesity epidemic that spurred some of us to pursue intensive research on the functional anatomy of the vagus nerve. As schematically depicted in Figure 1 , the vagus nerve with its sensory and motor fibers is one of the key players in reciprocal gut-brain communication.

The sympathetic nervous system and dorsal root spinal afferents are no doubt also important for reciprocal fast signal transmission between the gut and the brain, though we know much less of their potential implications in ingestive behavior and metabolic regulation. Spinal primary afferent neurons with cell bodies in dorsal root ganglia (DRGs) innervate the entire gastrointestinal tract and associated glands (pancreas, liver, gallbladder) as well as immune organs (lymph nodes, gut-associated lymphoid tissue, spleen).

Humoral factors secreted by the gut, and pituitary hormones discharged by the brain, additionally serve for slower communication with each other. However, it would be shortsighted to consider only direct connections between the gut and the brain, as 


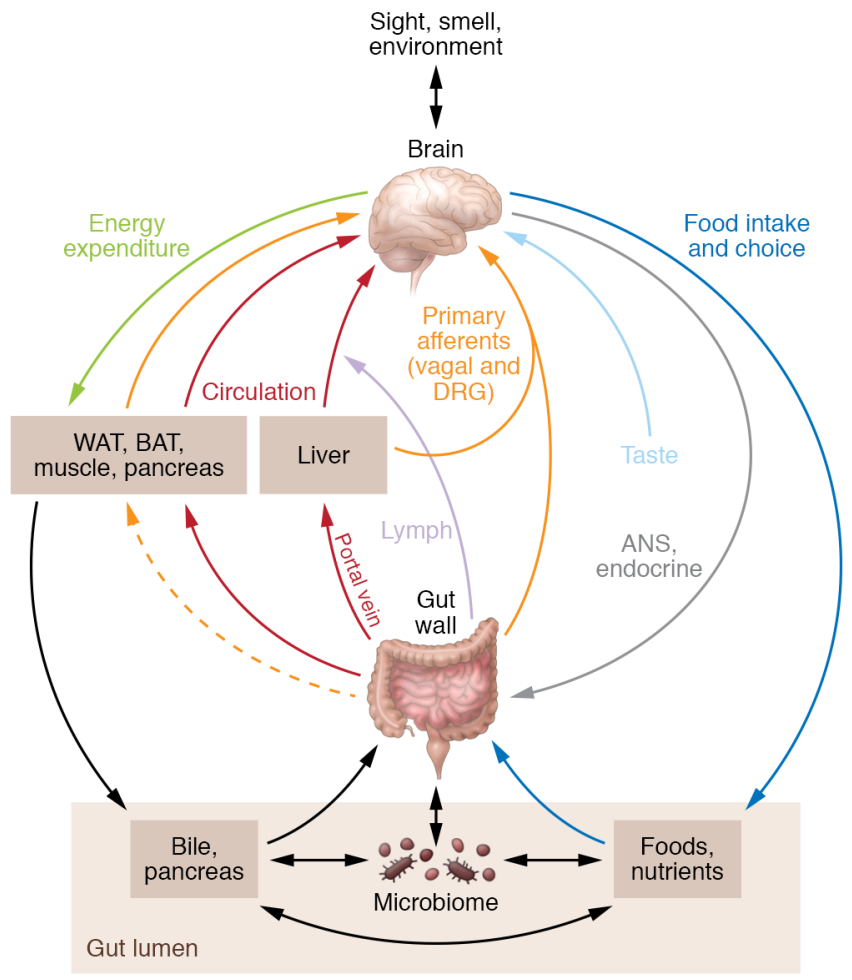

Figure 1. Schematic diagram showing potential gut-brain communication pathways relevant to obesity and metabolic diseases. See article text for details. BAT, brown adipose tissue; WAT, white adipose tissue; ANS, autonomic nervous system.

indirect neural and humoral signaling pathways via other organs are potentially also important (Figure 1). For eating behavior and metabolic regulation, the liver, adipose tissue, muscle, and pancreas, with their secretion of hormones and cytokines as well as their innervation by the autonomic nervous system and primary afferent neurons, are of particular interest. Besides hormones and cytokines, the recently discovered exosomes and microRNAs should also be considered for organ-to-organ communication (15, 16). Finally, bidirectional communication is further highlighted by the fact that the brain dictates how much and what kind of food is ingested (ingestive behavior), which subsequently determines how the gut and associated organs (e.g., microbiome, bile system, and pancreas) react and ultimately determines the type of feedback sent back to the brain and other organs (Figure 1).

\section{The role of gut-brain communication in eating behavior}

While earlier research focused mainly on mechanisms for signaling satiation, there is now much appreciation for signaling of appetition and food reward from the gut to the brain (17-23). The term "appetition" was coined by Sclafani and his colleagues based on the observation that the interaction of certain nutrients with the intestinal mucosa elicits more appetite, rather than less, as in satiation (22). We have long known the French proverb, "L'appétit vient en mangeant" (appetite comes with eating), referring to the fact that external cues such as sight, smell, and taste signaling the availability of delicious beneficial food stimulate appetite for and consumption of this food $(24,25)$. It turns out that such a feed- forward mechanism extends to the small intestine. By using non-nutritive saccharin-sweetened and differentially flavored solutions combined with intragastric or intraduodenal nutrient infusions, Sclafani and colleagues comprehensively demonstrated that animals learn to associate taste and flavor of ingested foods with its postingestive consequences $(25,26)$. This learning mechanism has two important consequences. First, the increased intake of the flavored solution when paired with intragastric glucose or lipids as compared with intragastric water indicates that intragastric nutrients act as positive reinforcers that can activate the brain reward system $(20,21)$. Second, this mechanism is fundamental for subsequent food choice and survival, as it allows acceptance of beneficial foods or rejection of harmful foods before any large amount is sampled and ingested. Identification of the signal transduction pathway from arrival in the gut to changing behavior in the brain is thus of great interest and has recently led to exciting new discoveries.

\section{The role of the vagus nerve}

Historical background on functional vagal anatomy. Based on the early premise that their activation leads to satiation and reduced energy intake, vagal afferents have attracted considerable attention. Before the availability of current molecular genetics-based neurobiology techniques, the vagal afferent system was thoroughly mapped using retrograde and anterograde labeling methods and electrical nerve recording. At least three fundamentally different types of terminal architectures have been demonstrated in both rats and mice. Intramuscular arrays are contained in the external muscular layers, particularly in the stomach wall, and are thought to represent slowly adapting stretch receptors $(27,28)$. Intraganglionic laminar endings (IGLEs) cover parts of myenteric ganglia that lie between the longitudinal and circular external muscle layers throughout the esophagus and gastrointestinal tract (29). Based on indirect evidence obtained from guinea pig stomach (30), they are thought to sense the level of tension, whether generated by passive tissue stretch or generated by active muscle contraction. Ultrastructural observations also support a mechanosensory function (31). Combined with recent immunohistochemical data, ultrastructural observations suggest additional chemosensory functions and complex interactions of IGLEs with myenteric neurons and calcitonin gene-related peptide-positive spinal afferents passing through myenteric ganglia (31-33), suggesting additional chemosensory functions. Finally, mucosal endings have been traced to the entire gastrointestinal tract, with the highest density in the villi and crypts of the proximal small intestine $(34,35)$. Originally thought to mediate nutrient-induced satiation signals, they now are implicated in nutrient-induced appetition signaling (36). In addition to the gut itself, the associated hepatic portal vein $(37,38)$ and pancreatic $\beta$ cells $(39,40)$ are also innervated by vagal afferents.

Assignment of specific functions to vagal afferent populations with different terminal architectures and different locations along the gastrointestinal tract has been hampered mostly by the inadequacy of tools limited to electrical stimulation and surgical or chemical interruption of the entire subdiaphragmatic vagal trunks or their main branches. Although more selective vagal branch manipulations have helped elucidate the viscerotopy of 
vagal afferent and efferent innervation (41-44), these studies are still nonspecific with respect to sensory versus motor fibers and to sensory or motor function of subpopulations. Clearly, many scientists were attracted to the subdiaphragmatic vagus nerves and branches because of relatively easy access. The common hepatic branch, which separates from the left (anterior) subdiaphragmatic trunk just beneath the diaphragm, is particularly easy to locate and manipulate, and it has received by far the greatest attention. There has been a steady stream of publications using surgical transection of the common hepatic branch of the vagus nerve as a tool to study the role of the vagus nerve in liver function (for recent examples, see refs. 45-54). Unfortunately, most of these analyses seem unaware of the projection targets of vagal afferent and efferent fibers in the common hepatic branch, as identified in neuronal tracing and multiorgan functional analyses with electrical stimulation $(37,41,55)$. These studies in rats have collectively demonstrated that a majority of vagal fibers passing through the (easily accessible) hepatic branch innervate targets in the proximal small intestine, pylorus, antrum, and pancreas, while a minority innervate the hepatic portal vein, bile ducts, and the liver hilum (37). That is, fibers in the common hepatic branch join the dense plexus surrounding the common hepatic artery, and the majority continue along the gastroduodenal artery. Only a minority of these fibers follow the hepatic artery proper toward the liver and portal vein (Figure 2). In recent studies addressing the benefit of preserving vagal pathways during cancer gastrectomy, similar findings were made in humans (56). Thus, cutting the common hepatic branch does a lot more than interrupt vagal motor input to the liver or vagal sensory output from the liver, and these potential collateral effects must be taken into consideration in interpreting such studies.

New generation of genetically guided vagal manipulations. Similar to the central nervous system, advances in genetics-based identification of molecularly distinct neurons have tremendously enriched our tool kit to study functional anatomy of the peripheral nervous system. First, with the use of reporter mice expressing brightly fluorescent proteins in all, or specific, populations of vagal sensory or motor neurons, the demanding and capricious anterograde tracer injections into the nodose ganglia or the dorsal motor nucleus could be circumvented, as well as often elaborate staining techniques (5762). In general, these studies in mice confirmed the distribution and terminal architecture of both vagal afferents and efferents reported earlier in rats using anterograde labeling techniques.

Second, transcriptional profiling of neurons contained in spatially compact areas such as the jugular, nodose, dorsal root, or myenteric plexus ganglia with single-cell RNA sequencing (scRNA-Seq) has provided comprehensive atlases of molecularly distinct clusters of neurons (62-66), suggesting that they represent function-specific populations. Specifically, and most relevant to this discussion, applying scRNA-Seq to individual handpicked neurons in the nodose ganglia labeled by injections of a retrograde tracer into different segments of the gastrointestinal tract and the hepatic portal vein further revealed the organization of vagal afferent innervation of abdominal organs (62). These studies demonstrated that each site was innervated by vagal sensory neurons belonging to different clusters and thus different functionality, but with more or less enrichment from one or more clus- ters. Given the specific organization of vagal motor outflow from the dorsal motor nucleus with respect to viscerotopy $(41,43,55$, 67-69) and function (70-72), similar scRNA-Seq analyses of functional and projection-specific vagal motor neurons in the dorsal motor nucleus are eagerly awaited.

Third, analysis of the effects of selective acute and chronic activation or inhibition of molecularly distinct subpopulations of vagal afferents on ingestive behavior and metabolic endpoints is well under way. Much of this research has focused on the mechanisms responsible for the post-oral detection of ingested nutrients, glucose and fat in particular, which provides the unconditioned stimulus for learning of food preferences as well as signals for appetition and satiation $(21,23,25,26,73)$. Thus, optogenetic silencing of a specific population of enteroendocrine cells (EECs) that make synaptic contacts with vagal afferent neurons in the proximal small intestine, so-called neuropod cells, abolishes the vagal afferent signal to intestinal sucrose and prevents mice from distinguishing non-nutritive sweeteners from nutritive sucrose $(74,75)$. Elaborate in vitro and in vivo studies further identified a mechanism by which luminal glucose is transported into the EECs selectively through sodium-glucose transporter-1 (SGLT1) (76), which leads to cell depolarization, the release of glutamate from the neuropods, and rapid activation of glutamate receptors on vagal afferent terminals (ref. 36 and Figure 2). While the specific vagal afferent population was not identified and therefore was not accessible for manipulation, another recent study used the PhosphoTRAP methodology to identify the vagal afferent neurons in the nodose ganglia that were activated by intestinal glucose but not the artificial sweetener acesulfame potassium (Ace-K) (77). In addition, these researchers identified the activated downstream neurons expressing proenkephalin in the nuclei tractus solitarii (NTS) (Figure 2). Importantly, silencing either the specific neuron populations in the nodose ganglia or the NTS abolished the ability of mice to learn a preference for intestinal glucose (77). In fact, optogenetic activation of these neurons while the mice were receiving differentially preferred intestinal stimuli led to a reversal of preferences, with the previously avoided stimulus now preferred (77).

What is most surprising is that selectively silencing these vagal pathways $(36,77)$ results in complete abolition of discriminatory nutrient preference learning. Given that such learning may be crucial for survival in the wild, this complete abolition is unexpected, as it would leave no room for any other signaling pathway to be involved in this fundamental behavior. One possible explanation is that it may be an artifact of the highly simplified situation with purified nutritive and non-nutritive solutions directly infused into the intestines, which is far from the normal task to discriminate among a multitude of food items. Thus, studies in more naturalistic environments with more complex food choices should be revealing. Also, given the extensive literature on humoral mediation of gut-to-brain nutrient signaling (e.g., refs. 78, 79), these recent findings suggest that different signaling pathways mediate different behavioral endpoints, such as satiation, appetition, food seeking, and reward generation.

It is also important in this regard to note that previous attempts using highly nonselective total subdiaphragmatic vagotomy or the slightly more selective subdiaphragmatic vagal deafferentation 


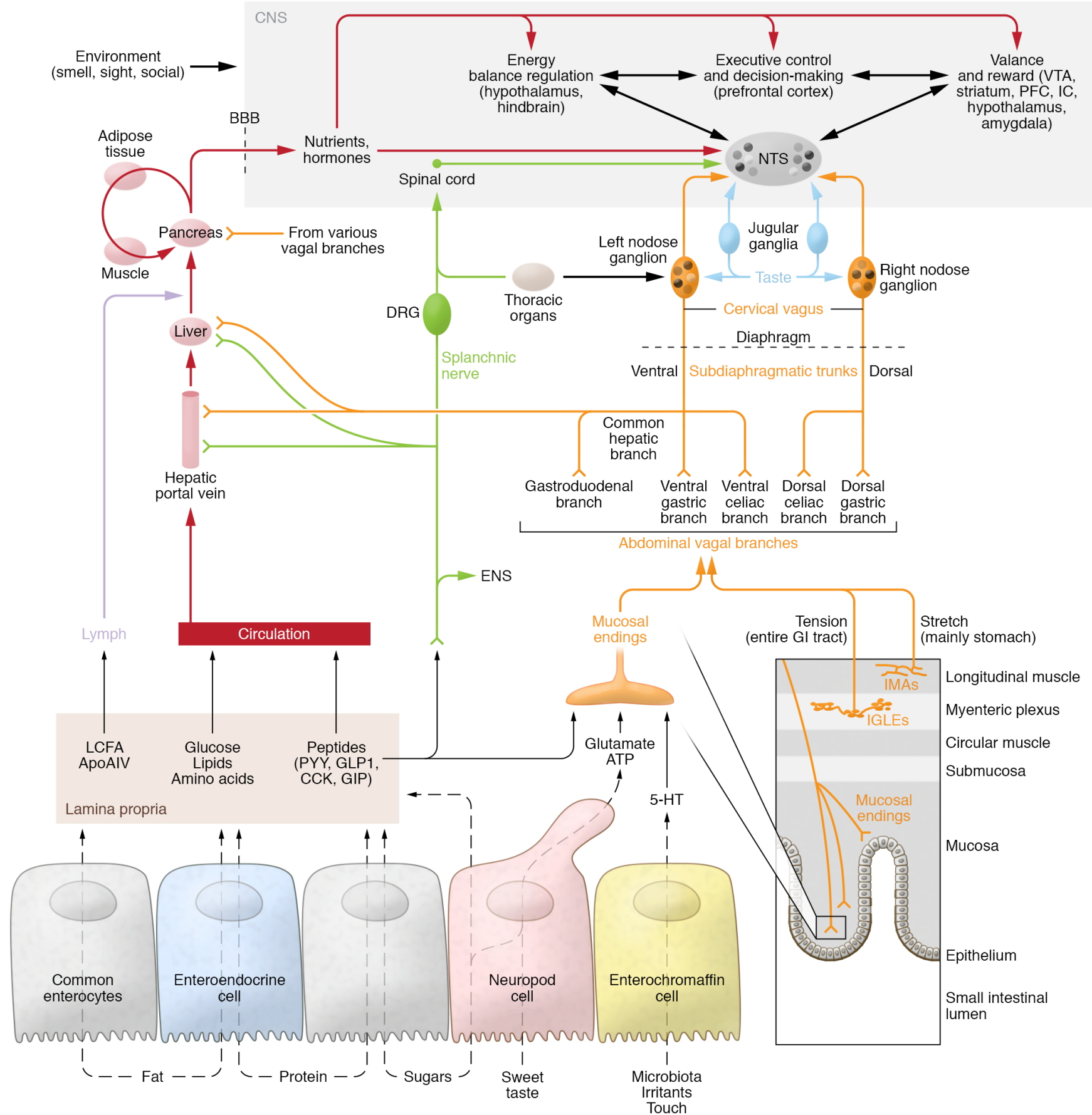

Figure 2. Gut-brain communication relevant to nutrient assimilation. Bottom: Transport mechanisms for dietary nutrients in small-intestinal epithelial cells and transduction mechanisms generating neural and humoral signals. Multistep pathways are shown using dashed lines. The volume and osmotic effects of ingested nutrients also interact with the muscular wall of the alimentary canal and can activate vagal stretch (intramuscular arrays [IMAs] mainly in the stomach) and tension receptors (intraganglionic laminar endings [IGLEs] throughout the gastrointestinal tract). Middle: Signals generated in vagal afferent terminals are projecting through several subdiaphragmatic branches (orange) to the nuclei tractus solitarii (NTS) via the nodose ganglia, which contain the neural cell bodies. Dorsal root afferents can also mediate signals to the brain (green), including the NTS via the spinal cord and spinosolitary tract. The lamina propria is innervated by enteric neurons, including intrinsic primary afferent neurons mediating mucosal signals to the enteric nervous system. Also note that nutrients and hormones in the portal hepatic vein and liver can potentially activate vagal and DRG afferents. Top: The NTS is a hub for sensory neural information originating from the gastrointestinal tract and the oral cavity as well as from humoral inputs via the area postrema and has extensive projections to many brain areas. These include areas involved in the control of food intake (satiation and hunger); homeostatic regulation of energy balance and body weight; the generation of food reward; and executive control and decision-making. Also note that nutrients and hormones taken up into the bloodstream (either directly or after transport through the lymphatic system) can eventually interact with sensors in specific areas of the brain and all other organs. VTA, ventral tegmental area; PFC, prefrontal cortex; IC, insular cortex; BBB, blood-brain barrier; ENS, enteric nervous system; LCFA, long-chin fatty acids; ApoAIV, apolipoprotein AIV; 5-HT, 5-hydroxytryptamine (serotonin). 
failed to implicate vagal afferents in nutrient preference learning (80-82), suggesting that at least some critical information is mediated from gut to brain by humoral signals. However, it must also be acknowledged that these nonspecific vagotomies (and similarly manipulations of dorsal root and sympathetic innervation by means of celiac/superior mesenteric ganglionectomy; refs. 81, 83) produce multiple side effects, particularly in the long term, that may occlude the true role of critical, small, and function-specific populations of sensory and/or motor neurons. Regeneration of surgically transected abdominal vagal afferents is another potential confounding problem (84).

\section{The role of other neural pathways}

Compared with the vagus nerve, sensory and sympathetic motor fibers innervating the abdominal organs via the spinal cord have received much less attention. This is at least in part due to the challenging anatomical configuration of sensory innervation by dorsal root ganglia (DRG) and the sympathetic nervous system (SNS). There is no "easy target" such as the common hepatic branch for the vagus, and researchers have targeted either the major splanchnic nerve $(85,86)$ or the celiac/superior mesenteric ganglion complex $(81,83)$. However, these manipulations are problematic, for the same reasons as are nonselective vagotomies, because there are likely many function-specific populations of SNS efferents. In particular, there are specific sympathetic pre-postganglionic neuron chains of motility-inhibiting, secretion-inhibiting, and vasoconstrictor neurons, which were, at their postganglionic level, defined by their peptide content (87). Also, instead of just two easily accessible nodose ganglia, there are many DRGs harboring a variety of transcriptionally defined subtypes (65) that are even more difficult to manipulate. To date, reporter mice with fluorescently labeled neurons and RNA-Seq have not been applied to the investigation of the innervation patterns in abdominal and other relevant organs, or to investigation of the potential roles of postganglionic SNS and DRG neurons in nutrient sensing and metabolic control. One recent study reported that the duodenal-glucose-induced drop in hypothalamic agouti-related peptide neuron activity was significantly attenuated in mice with prior celiac/superior mesenteric ganglionectomy, while the duodenal-fat-induced drop was attenuated in mice with subdiaphragmatic vagotomy (83), which may be explained by some of the functional differences between vagal and splanchnic glucosensors reported earlier (88). Whether these findings can be confirmed with more selective manipulations remains to be seen.

The potential of viscerofugal enteric neurons to affect food intake and glucose regulation was recently explored with chemogenetic activation of myenteric plexus neurons expressing cocaine- and amphetamine-regulated transcript (CART) located in the microbiota-rich regions, the distal ileum and colon, and projecting to the celiac/superior mesenteric ganglia (89). It was concluded that CART-expressing neurons can modulate blood glucose in a microbiota-dependent fashion via a polysynaptic pathway from the lower intestine to the celiac/superior mesenteric sympathetic ganglia and, in turn, to the pancreas (89). However, the suppressive effects on food intake, glucose tolerance, and insulin secretion were small and rather spurious, and the potential role of such a signaling pathway in normal physiology awaits further interventional approaches.

Clearly, comprehensive atlases of molecularly distinct, projection-specific postganglionic sympathetic neurons and DRG neurons would greatly facilitate further research into their potential roles in metabolic diseases.

\section{The role of gastrointestinal hormones and other factors}

As illustrated in Figure 2, a number of gastrointestinal hormones are secreted from EECs upon stimulation by various nutrients and other factors. Based on studies with systemic administration of such hormones and factors in vagotomized rodents, the generally held view was that most of these hormones are able to change brain function and food intake via both the bloodstream and vagal sensory neurons.

For example, glucagon-like peptide-1 (GLP-1) is produced by EECs located mainly, but not exclusively, in the distal bowel upon carbohydrate and protein absorption (78, 90). GLP-1 and its mechanisms of action have attracted the bulk of attention because stable GLP-1 analogs such as liraglutide and semaglutide are currently among the most effective pharmacological treatments to achieve remission of type 2 diabetes and reductions in food intake and body weight (91-93). In addition, increased GLP-1 signaling is among the leading candidates when it comes to explaining the beneficial effects of bariatric surgeries (94). Our understanding of intestinal GLP-1's exact signaling pathways has been complicated by its short half-life time in peripheral blood, as well as the presence of GLP-1-producing neurons in the NTS and their extensive projections to brain areas involved in energy homeostasis and glycemic control $(78,95)$. Even after extensive experimentation in a number of species, there remains considerable controversy as to the role of vagal afferents in the physiological and pharmacological effects of GLP-1 and its analogs on food intake and body weight regulation (ref. 95; for an in-depth discussion, see section 13 in ref. 78).

The recent genetics-guided approaches tend to support the conclusion that information from intestinal nutrient sensors does not depend on GLP-1 receptor-bearing (GLP-1R-bearing) vagal afferents. Surprisingly, GLP-1R is mainly expressed in vagal afferents forming IGLEs between the external muscle layers of the stomach and is only expressed in a small subset of vagal afferents innervating the intestinal villi $(61,62)$. The observation that selective optogenetic activation of GLP-1Rexpressing vagal afferents reduces short-term food intake (62) is consistent with the idea that gastric distention contributes to the satiation process and that circulating GLP-1 may modulate their sensitivity (96). However, as pointed out in a recent review (96), the small subset of GLP-1R-expressing vagal afferents innervating the intestinal villi could still be involved in the direct paracrine or synaptic (neuropods) activation of vagal afferents by GLP-1 released from EECs and its upstream effects on satiation via the NTS (95).

A fraction of bile acids escapes the enterohepatic circulation and "leaks" into the peripheral circulation. As bile acids are bona fide ligands for several receptors, this allows for central/peripheral actions of bile acids outside the gastrointestinal tract. Notably, lean animals with bile diversion surgery (to augment peripher- 
ally circulating bile acids) have TGR5-dependent reductions in cocaine-induced elevation of accumbal dopamine relative to controls, suggestive of a gut-brain hormonal circuit and potential for treatment for addiction (97).

One problem that arises with focusing on single hormones and factors, as is done in most reductionist experimental settings, is the likelihood that processes like satiation and appetition are regulated by the interaction and synergism between many factors. Although integration of afferent signals controlling food intake at all levels of the neuraxis has been recognized for a long time (e.g., see ref. 98), this issue has recently gained more attention. Thus, the combinatorial actions of GLP-1 and other gut hormones like CCK and PYY, whether mediated by vagal afferents or mediated through the bloodstream, are likely to exceed the individual effects (e.g., see refs. 99, 100), and this concept has found its way into the design of combinatorial polypharmacy for the treatment of metabolic diseases (101).

\section{Gut-brain communication in energy balance regulation and obesity}

Long-term energy balance is determined by energy intake, absorptive efficiency, and energy expenditure. Therefore, experimentally induced changes in eating do not necessarily translate into changes in body weight, or more specifically, overeating does not necessarily lead to obesity. This is particularly true for manipulations and challenges that change the satiation process, as changes in meal size are often compensated by changes in meal frequency, with total energy intake unchanged. Here we focus on the far fewer studies of gut-brain communication that provide clear evidence that it causes, prevents, or reverses obesity.

\section{Neural communication pathways}

Since vagal afferents have traditionally been mostly implicated in the satiation process, it is not surprising that little evidence for their role in body weight regulation has been provided. Earlier studies using indiscriminate surgical or chemical interruption of vagal afferents through subdiaphragmatic vagal deafferentation in rats or capsaicin treatment in rats and mice found small effects on meal patterns but no overt body weight phenotype (102). Similarly, silencing of vagal afferents using saporin-conjugated CCK injections into the nodose ganglia of rats eliminated CCK- and GLP-1-induced suppression of food intake but had apparently no effect on body weight (103). These observations are reminiscent of observations in rats with meal-contingent, exogenous CCK administration (104) demonstrating that increased meal size is compensated by decreased meal frequency to maintain normal body weight. Also, more selective vagal deafferentation procedures, such as the selective elimination of leptin receptors in vagal afferents in the Nav1.8/LepR $\mathrm{R}^{\mathrm{fl} / \mathrm{fl}}$ mouse (105) or knockdown of CART transcript in vagal afferent neurons in rats (106), result in a small increase of body weight and adiposity, with slightly increased nighttime food intake and no change in energy expenditure. However, diminished vagal afferent signaling after saporin-CCK injection into the nodose ganglia of rats on a highfat/high-sugar diet was clearly shown to contribute to excessive body weight gain and adiposity (107). Given that the sensitivity of gut vagal afferents is decreased in obese rodents (108), it will be important to further distinguish what comes first: obesity, or blunting of vagal afferent sensitivity (108).

The most recent genetics-guided selective manipulations of vagal afferents in mice that yielded specific deficits in nutrient preference learning were not tested for their long-term effects (36, $62,77)$. Specifically, it will be interesting to see whether chronic chemogenetic activation of vagal afferent neurons in the two mouse lines that showed significant acute suppression of food intake (Oxtr ${ }^{\text {Cre }}$ and Glp1r ${ }^{\mathrm{Cre}}$ mice; ref. 62) also reduces long-term food intake and body weight. Similarly, it will be interesting to see whether chronic activation of neuropod cells or their signaling capacity to vagal afferents (36) or proenkephalin-expressing NTS neurons (77) could be leveraged to suppress long-term food choice, caloric intake, and body weight.

In humans, electrical stimulation of the left cervical vagus nerve, a procedure used to treat refractory epilepsy and depression, has been reported to cause weight loss $(109,110)$ and increased energy expenditure through BAT thermogenesis (111). A report of reduced food craving upon vagus nerve stimulation (112) was, however, criticized as providing evidence not for reduced craving but rather for confused appetite in these depressed patients (113). A pilot clinical trial revealed beneficial effects on glucose metabolism also with transcutaneous stimulation of the left auricular branch of the vagus nerve (114). Although the vagal branches stimulated in these studies were not cut, it is assumed that the observed effects resulted mainly from activation of vagal afferents and the downstream brain areas (115), not vagal motor outflow. As vagal afferents are relayed by the NTS to various midbrain and forebrain sites, these beneficial effects may be generated at emotional and cognitive levels. However, a loop through the hypothalamus may link stimulation of vagal afferents to efferent autonomic and neuroendocrine channels. Thus, BAT thermogenesis via sympathetic nerves $(116,117)$, and possibly activation of efferent neurons of the dorsal vagal motor nucleus to the gut and pancreas, are conceivable. Interestingly, the opposite strategy, vagal blockade using high-frequency electrical stimulation of the subdiaphragmatic vagal trunks (vBloc Therapy, EnteroMedics Inc), has also been reported to result in meaningful weight loss (118), reminiscent of total subdiaphragmatic vagotomy recommended in the early days of surgical intervention for the treatment of obesity (119). For all these approaches, it will ultimately be important to identify and verify exactly what function-specific vagal afferents (or efferents) are activated or silenced, in order to generate coherent mechanistic explanations and design more selective treatments.

Finally, the antiinflammatory effects of electrical stimulation of vagal motor outflow in rodents have been widely publicized and its mechanisms discussed (for recent reviews, see refs. 120-122). To what extent the antiinflammatory reflex is linked to the pathogenesis of obesity, and whether it can be successfully translated to treat patients with obesity, remain to be elucidated (123).

\section{Humoral signaling by gut hormones and other factors}

As already discussed above, many gut hormones play important roles in the control of food intake and glycemic control. However, only GLP-1 agonists are currently marketed as effective antiobesity and/or antidiabetic drugs, with several others in early clinical trials $(92,93,124)$. Given our emphasis on vagal communication, we only 
briefly discuss the potential role of gut hormones and other factors directly acting on the brain, and the interested reader should consult the comprehensive recent review by Gimeno et al. (124).

Besides the classical gut hormones, bile acids are increasingly recognized for their actions not only in the gut and liver, but also in many other organs, including the brain, via the widely distributed bile acid receptors FXR and TGR5 (125). Importantly, the gutrestricted FXR agonist fexaramine potently attenuates dietinduced obesity and its associated inflammation and impairments in glucose metabolism in mice over a period of 5 weeks (117). The effect was attributed to increased brown fat thermogenesis and browning of white fat, but not decreased food intake. Furthermore, increased circulating levels of the intestinal hormone FGF15/19 and a shift in the bile acid profile, triggering changes in the microbiome and increased signaling through TGR5, were identified as potential mechanisms $(117,126)$. Although this would not imply gut-brain communication at first sight, it seems that the specific changes in bile acid profile and gut microbiota can affect glucose homeostasis via an obligatory relay in the dorsal vagal complex (127). In addition, bile acids can modulate GLP-1 secretion from EECs through a complex interaction between inhibitory FXR and stimulatory TGR5 effects (128-130). Aside from the bile acid-receptor interaction itself, bile acids stimulate secretion of FGF15/19 from enterocytes (131), which in turn may act in the brain to suppress food intake and body weight, and improve glucose homeostasis $(132,133)$.

\section{Gut microbiota}

The majority of studies on gut microbiota and obesity are cross-sectional and simply describe associations between specific microbes and obesity. Although such studies are helpful, they cannot provide evidence for causal relationships. Only interventional studies that directly or indirectly manipulate gut microbiota and their signaling pathways can demonstrate the necessity of gut microbiota for the regulation of body weight/adiposity.

The effects of prebiotics (foods that stimulate beneficial microbiota, e.g., inulins, fructo-oligosaccharides), probiotics (life microorganisms such as Lactobacillus and bifidobacteria), and synbiotics (synergistic combinations of pre- and probiotics) in patients with obesity and diabetes have been studied in many randomized placebo-controlled trials, with a number of meta-analyses available. Although most of these meta-analyses find small beneficial effects, they also emphasize the poor quality of evidence due to methodological variability and low sample numbers. The largest of these meta-analyses was based on 105 studies comprising a total of 6826 subjects with overweight or obesity (134), and reported mean improvements of $-0.94 \mathrm{~kg}$ in body weight and $-0.55 \mathrm{~kg} / \mathrm{m}^{2}$ in BMI with probiotic treatment for 3-12 weeks in overweight but not obese subjects. It also found small improvements in fasting blood glucose and glycated hemoglobin (HbA1c) in type 2 diabetics, as well as small improvements in alanine and aspartate aminotransferase in subjects with fatty liver disease (134).

The gold standard for demonstrating a causal role for the gut microbiome in obesity is microbiota transplantation or fecal matter transplantation (FMT). A recent meta-analysis of six placebocontrolled studies with a total of 154 subjects with obesity and dia- betes found that although mean $\mathrm{HbA1c}(-1.69 \mathrm{mmol} / \mathrm{L})$ was lower and HDL-cholesterol $(0.09 \mathrm{mmol} / \mathrm{L})$ was higher 2-6 weeks after healthy-donor compared with control FMT, there were no significant effects on body weight and BMI after 6-12 weeks (135). Thus, the great promise for gut microbiota manipulations and their resulting changes in gut-brain communication as a cure for obesity has not yet been realized.

\section{Gut-brain communication and bariatric surgery}

Bariatric surgery is currently the most effective treatment to achieve sustained weight loss in patients with obesity, and altered gut-brain communication resulting in reduced appetite is the most widely presumed mechanism. Despite intensive preclinical and clinical research, no single mechanism has yet been identified to account for the weight loss, but altered signaling by gut hormones to the brain, either directly or via vagal afferents, has been among the leading candidates. In a large cohort of patients with or without prior vagotomy, Roux-en-Y gastric bypass (RYGB) produced the same weight loss up to 5 years (136). In contrast, selective transection of the celiac vagal branches, which innervate mainly the small and large intestines including the Roux limb, significantly attenuated RYGB-induced weight loss and hypophagia in rats (137). Furthermore, in mice, eating a meal during the first 2 weeks after RYGB leads to exaggerated activation of the brainstem anorexia pathway including the NTS, lateral parabrachial nucleus, and central amygdala, each thought to receive crucial input from vagal afferents (138140). These observations are consistent with the idea that increased activity of vagal afferents mediates at least some of the early effects of RYGB on food intake and body weight, but further research with more selective sensory vagotomies will be necessary.

Because RYGB - as well as vertical sleeve gastrectomy (VSG) - greatly increases circulating plasma levels of GLP-1 and PYY, gut hormones known to decrease food intake, they were initially thought to play major roles in the beneficial effects on body weight $(94,141)$. However, subsequent studies in mouse models of bariatric surgery showed that RYGB-induced weight loss is similar in mice with deficient GLP-1R, PYY/Y2R, or combined GLP-1R/Y2R signaling compared with wild-type mice (142-145), suggesting that the critical signaling molecules have not yet been identified. Similarly, bile acid signaling through either TGR5 (146) or FXR (147) is not required for RYGB-induced weight loss, although both TGR5 signaling and FXR signaling play a role in improvements of glycemic control after RYGB (147) and VSG (148-150), and FXR signaling plays a role in VSG-induced weight loss (148). Overall, numerous studies testing single molecules or hormones have failed to identify the driving mechanisms of bariatric surgery. Alternatively, it is much more likely that bariatric surgery alters numerous processes that collectively result in satiety, weight loss, and other beneficial effects that contribute to sustained weight loss.

\section{Conclusions}

The vagus nerve is undoubtedly one of the most important links between the gut and the brain when it comes to ingestive behavior and metabolic regulation. The new generation of genetics-based neural manipulations, while fully confirming the distribution and structure, has prompted reassessment of the functional roles of vagal afferent fibers innervating the gut and associated organs. 
As molecularly distinct clusters of vagal afferent neurons likely reflect function-specific populations, their selective manipulation should continue to revolutionize their functional anatomy, and the traditional, nonselective vagal manipulations should be abandoned. Similar strategies should also be applied to spinal afferents and autonomic nervous system outflow via the vagus nerve and SNS. It is hoped that a more comprehensive understanding of neural and non-neural pathways of communication will extend the therapeutic repertoire to treat obesity and metabolic disease.

\section{Acknowledgment}

Research in the Berthoud laboratory has been partially funded by NIH grant DK047348.

Address correspondence to: Hans-Rudolf Berthoud, Neurobiology of Nutrition and Metabolism Department, Pennington Biomedical Research Center, Louisiana State University System, 6400 Perkins Road, Baton Rouge, Louisiana 70808, USA. Phone: 225.763.2688; Email: berthohr@pbrc.edu.
1. NCD Risk Factor Collaboration. Worldwide trends in body-mass index, underweight, overweight, and obesity from 1975 to 2016: a pooled analysis of 2416 population-based measurement studies in 128.9 million children, adolescents, and adults. Lancet. 2017;390(10113):2627-2642.

2. Geary N. Control-theory models of body-weight regulation and body-weight-regulatory appetite. Appetite. 2020;144:104440.

3. Levitsky DA. The non-regulation of food intake in humans: hope for reversing the epidemic of obesity. Physiol Behav. 2005;86(5):623-632.

4. Woods SC, et al. Food intake and the regulation of body weight. Annu Rev Psychol. 2020;51:255-277.

5. Krashes MJ, et al. Melanocortin-4 receptorregulated energy homeostasis. Nat Neurosci. 2016;19(2):206-219.

6. Branco T, et al. Near-perfect synaptic integration by Nav1.7 in hypothalamic neurons regulates body weight. Cell. 2016;165(7):1749-1761.

7. Speakman JR. If body fatness is under physiological regulation, then how come we have an obesity epidemic? Physiology. 2014;29(2):88-98.

8. Berthoud HR, et al. The obesity epidemic in the face of homeostatic body weight regulation: what went wrong and how can it be fixed? Physiol Behav. 2020;222:112959.

9. de Castro JM, Plunkett S. A general model of intake regulation. Neurosci Biobehav Rev. 2002;26(5):581-595.

10. Allender $\mathrm{S}$, et al. A community based systems diagram of obesity causes. PLoS One. 2015;10(7):e0129683.

11. Prentice A, Jebb S. Energy intake/physical activity interactions in the homeostasis of body weight regulation. Nutr Rev. 2004;62(7 pt 2):S98-S104.

12. Kuhn KGE. Galeni Opera Omnia. Leipzig: Car. Cnoblochii, 1821-1833; published 1881.

13. Cryan JF, Dinan TG. Mind-altering microorganisms: the impact of the gut microbiota on brain and behaviour. Nat Rev Neurosci. 2012;13(10):701-712.

14. Gibbs J, et al. Cholecystokinin elicits satiety in rats with open gastric fistulas. Nature. 1973;245(5424):323-325.

15. Castillo-Armengol J, et al. Inter-organ communication: a gatekeeper for metabolic health. $E M B O$ Rep. 2019;20(9):e47903.

16. Pardo F, et al. Extracellular vesicles in obesity and diabetes mellitus. Mol Aspects Med. 2018;60:81-91.

17. Berthoud HR. Vagal and hormonal gut-brain communication: from satiation to satisfaction. Neurogastroenterol Motil. 2008;20(suppl 1):64-72.

18. Shechter A, Schwartz GJ. Gut-brain nutrient sensing in food reward. Appetite. 2018;122:32-35.
19. De Araujo IE, et al. Elucidating the neural circuitry linking gastrointestinal fat sensing to action selection and reward. Paper presented at: Appetite Annual SSIB Meeting; July 28-August 2, 2013; New Orleans, Louisiana, USA. http://www. ssib.org/web/past_programs/SSIB_2013.pdf. Accessed March 31, 2021.

20. de Araujo IE, et al. 2020. Rethinking food reward. Annu Rev Psychol. 2020;71:139-164.

21. Han W, et al. A neural circuit for gut-induced reward. Cell. 2018;175(3):665-678.

22. Sclafani A. Gut-brain nutrient signaling. Appetition vs. satiation. Appetite. 2013;71:454-458.

23. Tellez LA, et al. A gut lipid messenger links excess dietary fat to dopamine deficiency. Science. 2013;341(6147):800-802.

24. Ferriday D, Brunstrom JM. 2011. 'I just can't help myself': effects of food-cue exposure in overweight and lean individuals. Int JObes (Lond). 2011;35:142-149.

25. Sclafani A. How food preferences are learned: laboratory animal models. Proc Nutr Soc. 1995;54(2):419-427.

26. Sclafani A, Ackroff K. Nutrient-conditioned intake stimulation does not require a distinctive flavor cue in rats. Appetite. 2020;154:104793.

27. Paintal AS. A study of gastric stretch receptors; their role in the peripheral mechanism of satiation of hunger and thirst. JPhysiol. 1954;126(2):255-270.

28. Phillips RJ, Powley TL. Tension and stretch receptors in gastrointestinal smooth muscle: re-evaluating vagal mechanoreceptor electrophysiology. Brain Res Brain Res Rev. 2000;34(1-2):1-26.

29. Berthoud HR, Neuhuber WL. Distribution and morphology of vagal afferents supplying the digestive system. In: Tache Y, Wingate DL, eds. Brain-Gut Interactions. CRC Press; 1994:43-66.

30. Zagorodnyuk VP, et al. Intraganglionic laminar endings are mechano-transduction sites of vagal tension receptors in the guinea-pig stomach. JPhysiol. 2001;534(pt 1):255-268.

31. Neuhuber WL. Sensory vagal innervation of the rat esophagus and cardia: a light and electron microscopic anterograde tracing study. JAuton Nerv Syst. 1987;20(3):243-255.

32. Horling $\mathrm{L}$, et al. Localization of receptors for calcitonin-gene-related peptide to intraganglionic laminar endings of the mouse esophagus: peripheral interaction between vagal and spinal afferents? Histochem Cell Biol. 2014;141(3):321-335.

33. Hubsch M, et al. Muscarinic acetylcholine receptors in the mouse esophagus: focus on intraganglionic laminar endings (IGLEs). Neurogastroenterol Motil. 2013;25(8):e560-e573.
34. Berthoud HR, et al. Vagal sensors in the rat duodenal mucosa: distribution and structure as revealed by in vivo DiI-tracing. Anat Embryol (Berl). 1995;191(3):203-212.

35. Powley TL, et al. Vagal afferent innervation of the proximal gastrointestinal tract mucosa: chemoreceptor and mechanoreceptor architecture. J Comp Neurol. 2011;519(4):644-660.

36. Buchanan KL, et al. A gut sensor for sugar preference [preprint]. https://doi. org /10.1101/2020.03.06.981365. Posted on bioRxiv March 8, 2020.

37. Berthoud HR, et al. An anterograde tracing study of the vagal innervation of rat liver, portal vein and biliary system. Anat Embryol (Berl). 1992;186(5):431-442.

38. Garcia-Luna C, et al. The medullary targets of neurally conveyed sensory information from the rat hepatic portal and superior mesenteric veins. eNeuro. 2021;8(1):ENEURO.0419-20.2021.

39. Makhmutova M, et al. Pancreatic $\beta$-cells communicate with vagal sensory neurons. Gastroenterology. 2020;160(3):875-888.

40. Neuhuber WL. Vagal afferent fibers almost exclusively innervate islets in the rat pancreas as demonstrated by anterograde tracing. JAuton Nerv Syst. 1989;29(1):13-18.

41. Berthoud HR, et al. Topography of efferent vagal innervation of the rat gastrointestinal tract. Am J Physiol. 1991;260(1 pt 2):R200-R207.

42. Berthoud HR, Neuhuber WL. Peripheral and central functional neuroanatomy of sensory and motor innervation of the portal-hepatic axis and biliary system. In: Haussinger D, Jungermann K, eds. Liver and Nervous System. Kluwer; 1998:17-33.

43. Berthoud HR, et al. Localization of vagal preganglionics that stimulate insulin and glucagon secretion. Am J Physiol. 1990;258(1 pt 2):R160-R168.

44. Berthoud HR, et al. Evidence for a role of the gastric, coeliac and hepatic branches in vagally stimulated insulin secretion in the rat. J Auton Nerv Syst. 1983;7(2):97-110.

45. Lorenzo-Martin LF, et al. Vagal afferents contribute to sympathoexcitation-driven metabolic dysfunctions. J Endocrinol. 2019;240(3):483-496.

46. Somann JP, et al. Characterization of plasma cytokine response to intraperitoneally administered LPS \& subdiaphragmatic branch vagus nerve stimulation in rat model. PLoS One. 2019;14(3):e0214317.

47. Holland J, et al. A brain-melanocortin-vagus axis mediates adipose tissue expansion independently of energy intake. Cell Rep. 2019;27(8):2399-2410.

48. Jo BG, et al. Vagal afferent fibers contribute to 
the anti-inflammatory reactions by vagus nerve stimulation in concanavalin A model of hepatitis in rats. Mol Med. 2020;26(1):119.

49. Hashimoto $\mathrm{N}$, et al. Involvement of the hepatic branch of the vagus nerve in the regulation of plasma adipokine levels in rats fed a highfructose diet. J Nutr Biochem. 2019;71:90-97.

50. Li Y, et al. Central Sfrp5 regulates hepatic glucose flux and VLDL-triglyceride secretion. Metabolism. 2020;103:154029.

51. Kurabayashi A, et al. Murine remote preconditioning increases glucose uptake and suppresses gluconeogenesis in hepatocytes via a brain-liver neurocircuit, leading to counteracting glucose intolerance. Diabetes Res Clin Pract. 2018;139:288-299.

52. Lopez-Soldado I, et al. Effects of hepatic glycogen on food intake and glucose homeostasis are mediated by the vagus nerve in mice. Diabetologia. 2017;60(6):1076-1083.

53. Izumi $\mathrm{T}$, et al. Vagus-macrophage-hepatocyte link promotes post-injury liver regeneration and whole-body survival through hepatic FoxM1 activation. Nat Commun. 2018;9(1):5300.

54. Kwon E, et al. Optogenetic stimulation of the liver-projecting melanocortinergic pathway promotes hepatic glucose production. Nat Commun. 2020;11(1):6295.

55. Berthoud HR, et al. Abdominal pathways and central origin of rat vagal fibers that stimulate gastric acid. Gastroenterology. 1991;100(3):627-637.

56 . Kong SH, et al. Intraoperative neurophysiologic testing of the perigastric vagus nerve branches to evaluate viability and signals along nerve pathways during gastrectomy. J Gastric Cancer. 2019;19(1):49-61.

57. Gautron L, et al. Genetic tracing of Nav1.8expressing vagal afferents in the mouse. J Comp Neurol. 2011;519(15):3085-3101.

58. Gautron L, et al. Melanocortin-4 receptor expression in different classes of spinal and vagal primary afferent neurons in the mouse. J Comp Neurol. 2012;520(17):3933-3948.

59. Gautron L, et al. Vagal innervation patterns following Roux-en-Y gastric bypass in the mouse. Int JObes (Lond). 2013;37(12):1603-1607.

60. Tsumori T, et al. Intrapancreatic ganglia neurons receive projection fibers from melanocortin-4 receptor-expressing neurons in the dorsal motor nucleus of the vagus nerve of the mouse. Brain Res. 2013;1537:132-142.

61. Williams EK, et al. Sensory neurons that detect stretch and nutrients in the digestive system. Cell. 2016;166(1):209-221.

62. Bai L, et al. Genetic identification of vagal sensory neurons that control feeding. Cell. 2019;179(5):1129-1143.

63. Kupari J, et al. An atlas of vagal sensory neurons and their molecular specialization. Cell Rep. 2019;27(8):2508-2523.

64. Morarach K, et al. Diversification of molecularly defined myenteric neuron classes revealed by single-cell RNA sequencing. Nat Neurosci. 2021;24(1):34-46

65. Usoskin D, et al. Unbiased classification of sensory neuron types by large-scale single-cell RNA sequencing. Nat Neurosci. 2015;18(1):145-153.

66. May-Zhang A, et al. Combinatorial transcription- al profiling of mouse and human enteric neurons identifies shared and disparate subtypes in situ. Gastroenterology. 2021;160(3):P755-P770.

67. Wang J, et al. Functional vagal input to chemically identified neurons in pancreatic ganglia as revealed by Fos expression. Am J Physiol. 1999;277(5):E958-E964.

68. Berthoud HR, et al. Vagal-enteric interface: vagal activation-induced expression of $\mathrm{c}$-Fos and p-CREB in neurons of the upper gastrointestinal tract and pancreas. Anat Rec. 2001;262(1):29-40.

69. Berthoud HR, Powley TL. Morphology and distribution of efferent vagal innervation of rat pancreas as revealed with anterograde transport of Dil. Brain Res. 1991;553(2):336-341.

70. Babic T, et al. Pancreatic insulin and exocrine secretion are under the modulatory control of distinct subpopulations of vagal motoneurones in the rat. J Physiol. 2012;590(15):3611-3622.

71. Travagli RA, Anselmi L. Vagal neurocircuitry and its influence on gastric motility. Nat Rev Gastroenterol Hepatol. 2016;13(7):389-401.

72. Berthoud HR. Morphological analysis of vagal input to gastrin releasing peptide and vasoactive intestinal peptide containing neurons in the rat glandular stomach. J Comp Neurol. 1996;370(1):61-70.

73. Qu T, et al. On the roles of the duodenum and the vagus nerve in learned nutrient preferences. Appetite. 2019;139:145-151.

74. Kaelberer MM, et al. A gut-brain neural circuit for nutrient sensory transduction. Science. 2018;361(6408):eaat5236.

75. Kaelberer MM, et al. Neuropod cells: the emerging biology of gut-brain sensory transduction. Annu Rev Neurosci. 2020;43:337-353.

76. Koepsell H. Glucose transporters in the small intestine in health and disease. Pflugers Arch. 2020;472(9):1207-1248.

77. Tan HE, et al. The gut-brain axis mediates sugar preference. Nature. 2020;580(7804):511-516.

78. Muller TD, et al. Glucagon-like peptide 1 (GLP-1). Mol Metab. 2019;30:72-130.

79. Yanagi S, et al. The homeostatic force of ghrelin. Cell Metab. 2018;27(4):786-804.

80. Sclafani A, Ackroff K. Capsaicin-induced visceral deafferentation does not attenuate flavor conditioning by intragastric fat infusions in mice. Physiol Behav. 2019;208:112586.

81. Sclafani A, et al. Selective effects of vagal deafferentation and celiac-superior mesenteric ganglionectomy on the reinforcing and satiating action of intestinal nutrients. Physiol Behav. 2003;78(2):285-294.

82. Sclafani A, Lucas F. Abdominal vagotomy does not block carbohydrate-conditioned flavor preferences in rats. Physiol Behav. 1996;60(2):447-453.

83. Goldstein N, et al. Hypothalamic detection of macronutrients via multiple gut-brain pathways. Cell Metab. 2020;33(3):S1550-687.e5.

84. Phillips RJ, et al. Long-term regeneration of abdominal vagus: efferents fail while afferents succeed. J Comp Neurol. 2003;455(2):222-237.

85. Deutsch JA, Jang Ahn S. The splanchnic nerve and food intake regulation. Behav Neural Biol. 1986;45(1):43-47.

86. Grundy D. Signalling the state of the digestive tract. Auton Neurosci. 2006;125(1-2):76-80.
87. Furness JB, et al. The enteric nervous system and gastrointestinal innervation: integrated local and central control. Adv Exp Med Biol. 2014;817:39-71.

88. Mei N, et al. Comparison between the properties of the vagal and splanchnic glucoreceptors of the small intestine. Involvement in insulin release. J Auton Nerv Syst. 1984;10(3-4):275-278.

89. Muller PA, et al. Microbiota-modulated CART enteric neurons autonomously regulate blood glucose. Science. 2020;370(6514):314-321.

90. Gribble FM, Reimann F. Function and mechanisms of enteroendocrine cells and gut hormones in metabolism. Nat Rev Endocrinol. 2019;15(4):226-237.

91. Wright EE Jr., Aroda VR. Clinical review of the efficacy and safety of oral semaglutide in patients with type 2 diabetes considered for injectable GLP-1 receptor agonist therapy or currently on insulin therapy. Postgrad Med. 2020;132(sup2):26-36.

92. Christou GA, et al. Semaglutide as a promising antiobesity drug. Obes Rev. 2019;20 (6):805-815

93. Wilding JPH, et al. Once-weekly semaglutide in adults with overweight or obesity. $\mathrm{N} \mathrm{Engl} \mathrm{JMed.}$ 2021;384(11):989.

94. le Roux CW, et al. Gut hormones as mediators of appetite and weight loss after Roux-en-Y gastric bypass. Ann Surg. 2007;246(5):780-785.

95. Brierley DI, et al. Central and peripheral GLP-1 systems independently suppress eating. Nat Metab. 2021;3(2):258-273.

96. Krieger JP. Intestinal glucagon-like peptide-1 effects on food intake: physiological relevance and emerging mechanisms. Peptides. 2020;131:170342.

97. Reddy IA, et al. Bile diversion, a bariatric surgery, and bile acid signaling reduce central cocaine reward. PLoS Biol. 2018;16(7):e2006682.

98. Berthoud HR. Multiple neural systems controlling food intake and body weight. Neurosci Biobehav Rev. 2002;26(4):393-428.

99. Kjaergaard M, et al. PYY(3-36) and exendin-4 reduce food intake and activate neuronal circuits in a synergistic manner in mice. Neuropeptides. 2019;73:89-95.

100.Roth E, et al. Behavioural and neurochemical mechanisms underpinning the feeding-suppressive effect of GLP-1/CCK combinatorial therapy. Mol Metab. 2021;43:101118.

101. Tschop MH, et al. Unimolecular polypharmacy for treatment of diabetes and obesity. Cell Metab. 2016;24(1):51-62.

102.Schwartz GJ, et al. Gut vagal afferent lesions increase meal size but do not block gastric preload-induced feeding suppression. Am J Physiol. 1999;276(6):R1623-R1629.

103. Diepenbroek C, et al. Validation and characterization of a novel method for selective vagal deafferentation of the gut. Am J Physiol Gastrointest Liver Physiol. 2017;313(4):G342-G352.

104.West DB, et al. Infusion of cholecystokinin between meals into free-feeding rats fails to prolong the intermeal interval. Physiol Behav. 1987;39(1):111-115

105. de Lartigue G, et al. Deletion of leptin signaling in vagal afferent neurons results in hyperphagia and obesity. Mol Metab. 2014;3(6):595-607.

106.Lee SJ, et al. Blunted vagal cocaine- and 
amphetamine-regulated transcript promotes hyperphagia and weight gain. Cell Rep. 2020;30(6):2028-2039.e4.

107. McDougle $\mathrm{M}$, et al. Intact vagal gut-brain signalling prevents hyperphagia and excessive weight gain in response to high-fat high-sugar diet. Acta Physiol (Oxf). 2020;231(3):e13530.

108.Page AJ. Vagal afferent dysfunction in obesity: cause or effect. J Physiol. 2016;594(1):5-6.

109. Pardo JV, et al. Weight loss during chronic, cervical vagus nerve stimulation in depressed patients with obesity: an observation. Int JObes (Lond). 2007;31(11):1756-1759.

110. Burneo JG, et al. Weight loss associated with vagus nerve stimulation. Neurology. 2002;59(3):463-464.

111. Vijgen $\mathrm{GH}$, et al. Vagus nerve stimulation increases energy expenditure: relation to brown adipose tissue activity. PLoS One. 2013;8(10):e77221.

112. Bodenlos JS, et al. Vagus nerve stimulation acutely alters food craving in adults with depression. Appetite. 2007;48(2):145-153.

113. Gibson EL, Mohiyeddini C. Vagus nerve stimulation confuses appetite: comment on Bodenlos et al. (2007). Appetite. 2008;51(1):223-225; discussion 226-230.

114. Huang F, et al. Effect of transcutaneous auricular vagus nerve stimulation on impaired glucose tolerance: a pilot randomized study. $B M C$ Complement Altern Med. 2014;14:203.

115. Conway CR, et al. Association of cerebral metabolic activity changes with vagus nerve stimulation antidepressant response in treatment-resistant depression. Brain Stimul. 2013;6(5):788-797.

116. Blouet C, Schwartz GJ. Duodenal lipid sensing activates vagal afferents to regulate non-shivering brown fat thermogenesis in rats. PLoS One. 2012;7(12):e51898.

117. Fang $S$, et al. Intestinal FXR agonism promotes adipose tissue browning and reduces obesity and insulin resistance. Nat Med. 2015;21(2):159-165.

118. Ikramuddin S, et al. Effect of reversible intermittent intra-abdominal vagal nerve blockade on morbid obesity: the ReCharge randomized clinical trial. JAMA. 2014;312(9):915-922.

119. Kral JG. Vagotomy as a treatment for morbid obesity. Surg Clin North Am. 1979;59(6):1131-1138.

120.Pavlov VA, Tracey KJ. The vagus nerve and the inflammatory reflex - linking immunity and metabolism. Nat Rev Endocrinol. 2012;8(12):743-754.

121. Martelli D, et al. The cholinergic anti-inflammatory pathway: a critical review. Auton Neurosci. 2014;182:65-69.

122. Berthoud HR, Neuhuber WL. Vagal mecha- nisms as neuromodulatory targets for the treatment of metabolic disease. Ann N Y Acad Sci. 2019;1454(1):42-55.

123. Chang EH, et al. Cholinergic control of inflammation, metabolic dysfunction, and cognitive impairment in obesity-associated disorders: mechanisms and novel therapeutic opportunities. Front Neurosci. 2019;13:263.

124. Gimeno RE, et al. Leveraging the gut to treat metabolic disease. Cell Metab. 2020;31(4):679-698.

125. Pournaras DJ, le Roux CW. Are bile acids the new gut hormones? Lessons from weight loss surgery models. Endocrinology. 2013;154(7):2255-2256.

126. Pathak $\mathrm{P}$, et al. Intestine farnesoid $\mathrm{X}$ receptor agonist and the gut microbiota activate G-protein bile acid receptor-1 signaling to improve metabolism. Hepatology. 2018;68(4):1574-1588.

127. Zhang SY, et al. FXR in the dorsal vagal complex is sufficient and necessary for upper small intestinal microbiome-mediated changes of TCDCA to alter insulin action in rats [published online October 21, 2020]. Gut. https://doi.org/10.1136/ gutjnl-2020-321757.

128. Trabelsi MS, et al. Farnesoid X receptor inhibits glucagon-like peptide- 1 production by enteroendocrine L cells. Nat Commun. 2015;6:7629.

129. Albaugh VL, et al. Role of bile acids and GLP-1 in mediating the metabolic improvements of bariatric surgery. Gastroenterology. 2019;156(4):1041-1051.

130. Thomas C, et al. TGR5-mediated bile acid sensing controls glucose homeostasis. Cell Metab. 2009;10(3):167-177.

131. Li S, et al. Cytoplasmic tyrosine phosphatase Shp2 coordinates hepatic regulation of bile acid and FGF15/19 signaling to repress bile acid synthesis. Cell Metab. 2014;20(2):320-332.

132. Liu S, et al. A gut-brain axis regulating glucose metabolism mediated by bile acids and competitive fibroblast growth factor actions at the hypothalamus. Mol Metab. 2018;8:37-50.

133. Ryan KK, et al. Fibroblast growth factor-19 action in the brain reduces food intake and body weight and improves glucose tolerance in male rats. Endocrinology. 2013;154(1):9-15.

134. Koutnikova $\mathrm{H}$, et al. Impact of bacterial probiotics on obesity, diabetes and non-alcoholic fatty liver disease related variables: a systematic review and meta-analysis of randomised controlled trials. BMJOpen. 2019;9(3):e017995.

135. Proenca IM, et al. Fecal microbiota transplantation improves metabolic syndrome parameters: systematic review with meta-analysis based on randomized clinical trials. Nutr Res. 2020;83:1-14.
136. Okafor PN, et al. Effect of vagotomy during Rouxen-Y gastric bypass surgery on weight loss outcomes. Obes Res Clin Pract. 2014;9(3):S1871-280.

137. Hao Z, et al. Vagal innervation of intestine contributes to weight loss After Roux-en-Y gastric bypass surgery in rats. Obes Surg. 2014;24(12):2145-2151.

138. Carter ME, et al. Genetic identification of a neural circuit that suppresses appetite. Nature. 2013;503(7474):111-114

139. Campos CA, et al. Parabrachial CGRP neurons control meal termination. Cell Metab. 2016;23(5):811-820.

140. Mumphrey MB, et al. Eating in mice with gastric bypass surgery causes exaggerated activation of brainstem anorexia circuit. Int JObes (Lond). 2016;40(6):921-928.

141. le Roux CW, et al. Gut hormone profiles following bariatric surgery favor an anorectic state, facilitate weight loss, and improve metabolic parameters. Ann Surg. 2006;243(1):108-114.

142. Boland B, et al. The PYY/Y2R-deficient mouse responds normally to high-fat diet and gastric bypass surgery. Nutrients. 2019;11(3):E585.

143. Boland BB, et al. 2019. Combined loss of GLP-1R and $\mathrm{Y} 2 \mathrm{R}$ does not alter progression of high-fat diet-induced obesity or response to RYGB surgery in mice. Mol Metab. 2019;25:64-72.

144. Mokadem M, et al. Effects of Roux-en-Y gastric bypass on energy and glucose homeostasis are preserved in two mouse models of functional glucagon-like peptide-1 deficiency. Mol Metab. 2014;3(2):191-201.

145. Ye J, et al. GLP-1 receptor signaling is not required for reduced body weight after RYGB in rodents. Am J Physiol Regul Integr Comp Physiol. 2014;306(5):R352-R362.

146. Hao Z, et al. Roux-en-Y gastric bypass surgeryinduced weight loss and metabolic improvements are similar in TGR5-deficient and wildtype mice. Obes Surg. 2018;28(10):3227-3236

147. Li K, et al. Farnesoid X receptor contributes to body weight-independent improvements in glycemic control after Roux-en-Y gastric bypass surgery in diet-induced obese mice. Mol Metab. 2020;37:100980

148. Ryan KK, et al. FXR is a molecular target for the effects of vertical sleeve gastrectomy. Nature. 2014;509(7499):183-188.

149. McGavigan AK, et al. TGR5 contributes to glucoregulatory improvements after vertical sleeve gastrectomy in mice. Gut. 2017;66(2):226-234.

150. Ding L, et al. Vertical sleeve gastrectomy activates GPBAR-1/TGR5 to sustain weight loss, improve fatty liver, and remit insulin resistance in mice. Hepatology. 2016;64(3):760-773. 